\title{
HAEMOPHAGOCYTIC LYMPHOHISTIOCYTOSIS IN PREGNANT AND POSTPARTUM WOMEN
}

DOI: 10.36740/WLek202009107

\author{
Aleksandra Obuchowska², Maciej Kamiński' , Żaneta Kimber-Trojnar' ${ }^{1}$, Paulina Grzesik², Arkadiusz Standyło², \\ Karolina Turżańska ${ }^{3}$, Bożena Leszczyńka-Gorzelak' \\ 'CHAIR AND DEPARTMENT OF OBSTETRICS AND PERINATOLOGY, MEDICAL UNIVERSITY OF LUBLIN, LUBLIN, POLAND \\ ${ }^{2} M E M B E R S$ OF SCIENTIFIC ASSOCIATION AT THE CHAIR AND DEPARTMENT OF OBSTETRICS AND PERINATOLOGY, MEDICAL UNIVERSITY OF LUBLIN, \\ LUBLIN, POLAND \\ ${ }^{3}$ CHAIR AND DEPARTMENT OF REHABILITATION AND ORTHOPAEDICS, MEDICAL UNIVERSITY OF LUBLIN, LUBLIN, POLAND
}

\begin{abstract}
Introduction: Haemophagocytic lymphohistiocytosis $(\mathrm{HLH})$ is an extremely rare, life-threatening disease, caused by uncontrolled activation of lymphocytes $T$ and macrophages This situation leads to cytokine storm, infiltration and internal organs failure. HLH can be categorised into either primary (familiar) or secondary which may be associated with infections, immunodeficiency syndromes, autoimmune diseases and malignancy. The secondary HLH is difficult to diagnose due to nonspecific symptoms and complicated differential diagnostics.

The aim: To conduct a comparative analysis of pregnant and puerperal patients diagnosed with HLH.

Material and methods: Review of available literature on haemophagocytic lymphohistiocytosis during pregnancy and the puerperium

Results: Review of the latest literature shows that HLH can occur at any time during pregnancy and in the puerperium. Symptoms of the disease are non-specific: fever not responding to antibiotic therapy, sometimes hectic, hepatosplenomegaly, swelling, lymphadenopathy, disseminated intravascular coagulation, multi-organ failure and death. In laboratory tests, worsening bicytopenia or pancytopenia, increasing indicators of organ damage, hypertriglyceridemia, hypofibrinogenemia and abnormally high serum ferritin levels are observed.

Conclusions: $\mathrm{HLH}$, due to non-specific symptoms and rarity, is often overlooked in the diagnostic process. Due to the high mortality and morbidity rates of $\mathrm{HLH}$ during pregnancy for mother and foetus, timely diagnosis and the inclusion of specialist treatment are particularly important. An interdisciplinary approach to the patient is necessary to make an accurate diagnosis. The assessment of serum ferritin concentrations facilitates diagnosis. The bone marrow is essential to diagnosis and should be performed as early as possible.
\end{abstract}

KEY WORDS: haemophagocytic lymphohistiocytosis, hectic fever, pregnancy, puerperium, hyperferritinemia

Wiad Lek. 2020;73(9 p. I):1844-1847

\section{INTRODUCTION}

Haemophagocytic lymphohistiocytosis (HLH) is an extremely rare, hyperinflammatory clinical syndrome in which lymphocytes $\mathrm{T}$, macrophages and natural killer (NK) cells play an essential role. Uncontrolled accumulation of inflammatory cells which cause cytokine storm, may be a reason of multiple organ failure and can be potentially fatal. HLH progresses rapidly and has a high mortality rate despite appropriate management. HLH can be categorised into either primary (familiar) or secondary which may be associated with Epstein-Barr virus infection (53$90 \%)$, hematologic malignancy (13-22\%), rheumatologic illness (11\%), immunodeficiency syndromes, autoimmune diseases and drugs. Idiopathic cases represent $23-26 \%$ of HLH [1]. HLH in adults, mainly occurs as the secondary form. Pregnancy is a physiological process, that is why this syndrome is rare in it. However, case studies of pregnant and postpartum women with HLH are available in the literature.

\section{MATERIAL AND METHODS}

Review of available literature on haemophagocytic lymphohistiocytosis during pregnancy and the puerperium.

\section{THE AIM}

The purpose of the study was to conduct a comparative analysis of pregnant and puerperal patients diagnosed with $\mathrm{HLH}$, which is a very rare and often overlooked disease, that leads to death without rapid diagnosis and specialized treatment.

\section{REVIEW AND DISCUSSION}

From the literature review, it can be seen that the haemophagocytic syndrome can occur at any time during pregnancy (10-38 weeks) and in the puerperium.

Pregnant women represent an immunologically unique population because, the maternal immune system is regulated to protect the foetus from immunological recognition 
and rejection. Uterine natural killer ( $\mathrm{uNK}$ ) cells, immature dendritic cells (iDCs), T cells and macrophages contribute to modulate the uterine environment to sustain a successful pregnancy $[2,3]$. Dysregulated immune system during pregnancy and childbirth may affect the occurrence of HLH syndrome during this period in the group of generally healthy women without chronic diseases and infections.

The secondary HLH is more difficult to diagnose than primary type because of ambiguous symptoms and complicated differential diagnosis. Differentiation with other diseases such as infection, sepsis, acute fatty liver of pregnancy, acute cholecystitis, Still disease, HELLP syndrome, aplastic anaemia is necessary.

The HLH Study Group of the Histiocyte Society published the first diagnostic guidelines in 1991 [4]. Presently, the recognition is based on HLH-2004 diagnosis criteria [5]. The HLH diagnosis can be established if a molecular diagnosis consistent with HLH or diagnostic criteria for HLH are fulfilled, i.e. five of the eight following criteria:

- splenomegaly,

- fever,

- cytopenia (affecting two or more of three lineages in the peripheral blood),

- hypofibrinogenemia and/or hypertriglyceridemia,

- elevated ferritin,

- haemophagocytosis in bone marrow/spleen/lymph nodes,

- low or absent natural killer (NK)-cell activity

- elevated soluble CD25 (interleukin [IL]-2 receptor) [6, 7]. Fardet et al. developed and validated a scoring system (HScore) which can be used to estimate an individual's risk of having reactive haemophagocytic syndrome [8]. The Park's study showed that the median time from the first visit to the beginning of treatment equals 5.5 days [1].

The bone marrow biopsy is essential to diagnose and should be performed as early as possible, sometimes more than once. In accordance to the published HLH case studies the first bone marrow biopsy results may be negative, but the second time confirms the diagnosis [6]. However, lack of haemophagocytosis in the bone marrow does not exclude the diagnosis of HLH. This criterium can be demonstrated, for example, in liver biopsies [9].

Symptoms of the disease are usually uncharacteristic. Typical findings of HLH include fever (which is not responding to antibiotic therapy and sometimes hectic), hepatosplenomegaly, skin lesions, oedema, and lymphadenopathy. Fever occurs in most diagnosed cases of HLH $(95,7 \%)$ [1]. Laboratory tests can reveal bicytopenia or pancytopenia, hypertriglyceridemia, hypofibrinogenemia, high liver damage rates, and high inflammation indicators. The most characteristic symptom is hyperferritinemia. It should be emphasized that low serum levels of ferritin exclude the HLH diagnosis.

In a majority of cases the initial symptoms include fever lasting from several days to several months and cough or jaundice. Song et al. presented patients diagnosed with HLH in the postpartum period and most of them had a fever which started a few days after delivery (from 1 to 20 days) [2]. Frequently it lasted several months before or after delivery. Night sweating, fatigue and shivering also periodically occurred in this group.

Interestingly, it was noticed that cytopenia was less common in the postpartum women with HLH. Furthermore, $\mathrm{HLH}$ in the postpartum period is usually associated with the infectious aetiology. Fever is very common in this period. Nonetheless, the HLH diagnostics may be more difficult after childbirth. It is worth paying attention that the liver dysfunction (sometimes recurrent) occurred in almost all cases of HLH in the puerperium, whereas acute hepatitis was described in $40 \%$ cases of pregnant patients $[2,9]$. The jaundice was often observed as an accompanying symptom. In many cases computed tomography (CT) scans and liver biopsies showed a possible fatty liver. After remission of $\mathrm{HLH}$, the liver function usually returns to normal [2]. However, recurrent liver dysfunction after a month of remission has also been reported [2]. Fortunately, a condition of this postpartum woman improved after liver protection therapy [2].

HLH was often associated with infection in pregnancy including Epstein-Barr virus (EBV), human immunodeficiency viruses (HIV), Cytomegalovirus (CMV), herpes simplex virus (HSV), and parvovirus B19.

Song et al. presented a pregnant patient with a fever for 10 days at 24 weeks gestation [2]. Because of unreasonable abdominal distension, enlarged liver and splenomegaly, the pregnancy was terminated. Despite this procedure the patient suffered from fever, jaundice and pancytopenia. Liver enzymes, triglycerides (TGs), and ferritin levels were elevated. HLH caused by EBV diagnosis was based on bone marrow examination and EBV DNA blood test [2]. Because of pneumonia, the doses of drugs in the HLH-94 protocol (which includes dexamethasone, etoposide, and cyclosporin A) were reduced [2]. However, the infection progressed and the patient died of multiorgan failure [2].

Yildiz et al. described a course of HLH in a pregnant patient with HIV infection in the third trimester. She suffered from itchiness and jaundice. The liver function was abnormal and levels of total bilirubin, alanine aminotransferase (ALT), aspartate aminotransferase (AST) and lactate dehydrogenase (LDH) were elevated. Haemoglobin concentration was under the reference ranges. The serum level of ferritin was elevated at $853 \mu \mathrm{g} / \mathrm{L}$. She was diagnosed with acute hepatitis inducted by viral infection or medicaments. Therefore, the antiretroviral treatment was stopped. After an emergency caesarean section, a high fever not responding to antibiotic therapy appeared. After 3 days, the concentration of ferritin increased to $10543 \mu \mathrm{g} / \mathrm{L}$ and TG level was 466 $\mathrm{mg} / \mathrm{dL}$. Finally, a liver biopsy was performed and features of HLH were demonstrated. This was the first patient with the HLH diagnosis based on the liver biopsy. The woman was treated with intravenous methylprednisolone $(80 \mathrm{mg}$ daily) for a week, then the drug was administered orally. Her clinical condition improved after 3 days of therapy and no other immunosuppressive agent was added [9].

The patient suffering from fever for 2 months (started 10 days after delivery), night sweating, fatigue and breast 
tenderness was described in 2019 [2]. She had enlarged mandibular lymph nodes and spleen. Laboratory abnormalities included pancytopenia and elevated concentrations of ALT, AST, TG, and ferritin. After determining the NK cell activity and sCD25 level, the diagnosis of HLH was confirmed. Doxorubicin, etoposide and methylprednisolone were used. No disease remission was achieved. Three months later the fever reappeared and the leishmania test was positive. This time she was treated with previous medications. Sodium gluconate was added to eliminate the parasites. After this treatment, the patient achieved the complete remission [2].

HLH cases have also been published in patients suffering from autoimmune diseases like systemic lupus erythematosus (SLE), Still's disease, and autoimmune haemolytic anaemia [10-12].

Song et al. described also a patient diagnosed with central nervous system-HLH (CNS-HLH) [2]. Patient presented a fever 3 days after caesarean section, right upper quadrant pain and palpable liver. She was initially diagnosed with acute cholecystitis and unsuccessfully treated with antibiotics. White blood cells (WBC) and haemoglobin were decreased. Liver enzymes, TG, ferritin and sCD25 levels were elevated. Fibrinogen was decreased. HLH was diagnosed and the HLH-94 therapy was initiated. Two weeks after treatment, the patient was diagnosed with central nervous system disorders, such as consciousness and speech disturbances as well as urinary and fecal incontinence. She was diagnosed with CNS-HLH and intrathecal injections of methotrexate and dexamethasone were administrated, but patient died due to multiorgan failure [2].

HLH secondary to NK/T cells lymphoma was described in 2017 [13]. The patient at 30 weeks of pregnancy suffered from the abdominal pain, splenomegaly, fever over $39^{\circ} \mathrm{C}$, pancytopenia, elevated ferritin, ALT, AST and LDH. In spite of the caesarean section the patient's condition was getting worse and disseminated intravascular coagulation (DIC) occurred. Test for EBV was positive and histopathological examination of the removed spleen showed an invasive NK/T-cell lymphoma and HLH. Despite the therapy with dexamethasone, etoposide and rituximab, the patient died [13].

In spite of extensive diagnostics, the aetiology of many cases of the secondary HLH are unknown [2]. There are also no clear guidelines for the treatment in the group of pregnant and postpartum women. The causal treatment starts according to the HLH-2004 protocol consisted of initial therapy with dexamethasone $10 \mathrm{mg} / \mathrm{m} 2$ per day, etoposide $150 \mathrm{mg} / \mathrm{m}^{2}$ twice a week and cyclosporine followed by dexamethasone intravenous pulses [5]. Steroids are used in the first line of treatment. The use of etoposide, which is a potential teratogenic drug, is controversial in the case of pregnant women. For this reason, it is better to use steroids at first line therapy and intravenous immunoglobulins or cyclosporine in steroids-resistant cases [9].

CNS-HLH is associated with a poor prognosis. It indicates the late stage of the disease [2]. The cerebral involvement may cause severe and irreversible damage. In the case of CNS-HLH, it is better to use dexamethasone because this steroid penetrates the blood-brain barrier better than prednisolone [3].

Park et al. indicate that initial serum fibrinogen concentration of more than $166 \mathrm{mg} / \mathrm{dL}$ is associated with better patient survival [1]. Whereas no correlation was found between the rate of decline of serum ferritin level and patient survival. The low histiocyte proportion (less than 5.6\%) in bone marrow and early initiation of treatment (less than 5.5 days) tends to correlate with favourable outcomes [1]. Therefore, earlier and quick HLH diagnosis is necessary for pregnant women and reduces the mortality rate.

The EBV infection is a factor for poor prognosis in the postpartum women [2]. In studies on a group of adult patients, the most common cause of HLH was EBV. The median follow-up period was 21 months. The total remission was observed in $40 \%$ of cases, whereas $10 \%$ of patients had the partial remission [1]. Other studies performed on adult people suggested that patients with EBV infection-associated HLH had good outcomes and an equal or even better prognosis than familial HLH in children [14]. Imashuku et al. in their study revealed that early etoposide treatment was effective in the treatment of EBV-HLH (the 2.5-year survival of $85.7 \pm 13.2 \%$ in the early etoposide-treated patients, compared to $10.3 \pm 9.4 \%$ in the remaining patients) [15]. The possibility of using an allogeneic hematopoietic stem cell transplantation has been noticed in some cases such as EBV-HLH with encephalitis or familiar HLH $[7,16]$.

\section{CONCLUSIONS}

HLH during pregnancy and the postpartum period represents a rare type of secondary HLH. Due to non-specific symptoms and uncommon occurrence, this disease is often overlooked in the diagnostic process. Increased awareness of HLH possibilities during pregnancy and also in the postpartum period is essential for early diagnosis and for timely treatment. HLH should be considered in pregnant and puerperal women suffering from fever not responding to antibiotic therapy, cytopenia, hepatitis and hyperferritinemia. Determining the level of ferritin can significantly contribute to an accurate diagnosis. The liver dysfunction was found in almost all cases. Timely diagnosis and inclusion of treatment are vital for the health and life of mothers and their children. The standard therapy with HLH-2004 is effective in the treatment of patients. It is important, if possible, to find the cause of the disease and to apply the causal treatment. It should be also highlighted that the period of pregnancy and the puerperium may predispose and extend the disclosure of various immune disorders. Due to greater awareness of the disease, diagnosis of HLH and patient survival have increased in recent years.

\section{REFERENCES}

1. Park H, Kim D, Lee J et al. Clinical features of adult patients with secondary hemophagocytic lymphohistiocytosis from causes other than lymphoma: an analysis of treatment outcome and prognostic factors. Ann Hematol. 2012;91(6):897-904. doi:10.1007/500277-011-1380-3. 
2. Song Y, Wang JS, Wang YN et.al. Hemophagocytic Lymphohistiocytosis during the Postpartum Stage of Pregnancy: A Report of Eight Cases. Acta Haematol. 2019;141(1):55-60. doi:10.1159/000493879.

3. PrabhuDas M, Bonney E, Caron K et al. Immune mechanisms at the maternal-fetal interface: perspectives and challenges. Nat Immunol. 2015;16(4):328-334. doi:10.1038/ni.3131.

4. Henter J, Elinder G, Ost A. Diagnostic guidelines for hemophagocytic lymphohistiocytosis. The FHL Study Group of the Histiocyte Society. Semin Oncol. 1991;18(1):29-33.

5. Henter J, Horne AC, Aricó M et al. HLH-2004: Diagnostic and therapeutic guidelines for hemophagocytic lymphohistiocytosis. Pediatr Blood Cancer. 2007;48(2):124-131.

6. Cheng J, Niu J, Wang Y et al. Hemophagocytic lymphohistiocytosis in pregnancy: a case report and review of the literature. J Obstet Gynaecol. 2020;40(2):153-159. doi:10.1080/01443615.2019.1601168.

7. Samra B, Yasmin M, Arnaout S et al. Idiopathic Hemophagocytic Lymphohistiocytosis During Pregnancy Treated with Steroids. Hematol Rep. 2015;7(3):6100. doi:10.4081/hr.2015.6100.

8. Fardet L, Galicier L, Lambotte 0 et al. Development and validation of the HScore, a score for the diagnosis of reactive hemophagocytic syndrome. Arthritis Rheumatol. 2014;66(9):2613-2620. doi:10.1002/art.38690.

9. Yildiz H, Vandercam B, Thissen X et al. Hepatitis during pregnancy: A case of hemophagocytic lymphohistiocytosis. Clin Res Hepatol Gastroenterol. 2018;42(3):e49-e55. doi:10.1016/j.clinre.2017.10.007.

10. Pérard L, Costedoat-Chalumeau N, Limal $\mathrm{N}$ et al. Hemophagocytic syndrome in a pregnant patient with systemic lupus erythematosus, complicated with preeclampsia and cerebral hemorrhage. Ann Hematol. 2007;86(7):541-544. doi:10.1007/s00277-007-0277-7.

11. Teng $C L$, Hwang GY, Lee BJ et al. Pregnancy-induced hemophagocytic lymphohistiocytosis combined with autoimmune hemolytic anemia. J Chin Med Assoc. 2009;72(3):156-159. doi:10.1016/S17264901(09)70043-7.

12. Dunn T, Cho M, Medeiros B et al. Hemophagocytic lymphohistiocytosis in pregnancy: a case report and review of treatment options. Hematology. 2012;17(6):325-328. doi:10.1179/1607845412Y.0000000007.

13. He M, Jia J, Zhang J et al. Pregnancy-associated hemophagocytic lymphohistiocytosis secondary to NK/T cells lymphoma: A case report and literature review. Medicine (Baltimore). 2017;96(47):e8628. doi:10.1097/MD.0000000000008628.

14. Sovinz P, Schwinger W, Lackner H et al. Severe epstein-barr virus encephalitis with hemophagocytic syndrome: rapid clearance of virus following allogeneic hematopoietic stem cell transplantation from a seropositive donor. Pediatr Infect Dis J. 2010;29(6):553-556. doi: 10.1097/INF.0b013e3181d1de1d.
15. Imashuku S, Teramura T, Tauchi H et al. Longitudinal follow-up of patients with Epstein-Barr virus-associated hemophagocytic lymphohistiocytosis. Haematologica. 2004;89(2):183-188.

16. Jabado N, de Graeff-Meeder ER, Cavazzana-Calvo M et al. Treatment of familial hemophagocytic lymphohistiocytosis with bone marrow transplantation from HLA genetically nonidentical donors. Blood. 1997;90(12):4743-4748.

\section{ORCID and contributionship:}

Aleksandra Obuchowska: 0000-0003-0464-2695 A,B,D,F

Maciej Kamiński: 0000-0002-2164-6762 A,B,D,F

Żaneta Kimber Trojnar: 0000-0001-7295-0409 B,D,F

Doctor Paulina Grzesik: 0000-0002-8033-1293 ${ }^{D}$

Student Arkadiusz Standyło: 0000-0002-5154-4759 D,F

Karolina Turżańska: 0000-0001-7359-6922 ${ }^{D}$

Bożena Leszczyńska Gorzelak: ORCID: 0000-0002-0221-1982 A,B,D,F

\section{Conflict of interest}

Authors declare no conflict of interest

\section{CORRESPONDING AUTHOR Maciej Kamiński,}

Chair and Department of Obstetrics and Perinatology, Medical University of Lublin, Poland Jaczewskiego str. 8, 20-954, Lublin, Poland; e-mail:dr.kaminski1993@gmail.com

Received: 06.07.2020

Accepted: 07.09 .2020

\footnotetext{
A - Work concept and design, B - Data collection and analysis, C - Responsibility for statistical analysis, D-Writing the article, $\mathbf{E}$-Critical review, $\mathbf{F}$ - Final approval of the article
} 